\title{
Channel Code Division Multiple Access and its Multilevel Structured LDPC Based Instantiation
}

\author{
Nicholas Bonello, Rong Zhang, Sheng Chen, and Lajos Hanzo \\ School of ECS, University of Southampton, SO17 1BJ, United Kingdom. \\ Email: $\{$ nb06r,rz05r,sqc,lh\} @ecs.soton.ac.uk, http://www-mobile.ecs.soton.ac.uk
}

\begin{abstract}
In this paper, we introduce and outline the concept of Channel Code Division Multiple Access (CCDMA) using a design example based on the recently proposed Multilevel Structured (MLS) LDPC codes. We succeeded in making the memory requirements of the multi-user transceiver to become practically independent of the total number of users supported by the system as well as ascertain that each user benefits from the same Quality of Service (QoS). Finally, we will demonstrate that despite their beneficial compact structure, the proposed MLS LDPC codes do not suffer from any Bit Error Ratio (BER) or Block Error Ratio (BLER) performance degradation, when compared to an otherwise identical benchmarker scheme using significantly more complex LDPC codes having pseudo-random parity-check matrices.
\end{abstract}

\section{INTRODUCTION}

The concept of generalized Code Division Multiple Access (CDMA) may be defined as a multiple access scheme, which separates the users in the code domain, whilst allowing them to share the same time and frequency resources. A traditional way of generating the user-specific codes is by employing distinct spreading codes, as in the well-known Direct Sequence (DS)-CDMA [1] scheme. Another possibility is to distinguish between users using user-specific channel codes, which is reminiscent of the concept of Trellis Coded Multiple Access (TCMA) [2] and Interleave Division Multiple Access (IDMA) [3]. In the former, the separation of the users is achieved by the unique combination of user-specific generator polynomials (GP) combined with bit-to-symbol mapping schemes and interleavers, whilst the latter employs user-specific interleavers, which may be regarded as rate-one channel codes. Hence we will jointly refer to these schemes using the generic terminology of Channel Code Division Multiple Access (CCDMA).

Typically, a relatively short code constraint length is favored in TCMA systems in order to attain a reasonably low decoding complexity. Naturally, this reduces both the number of GPs and the number of users supported. It reduces the probability of encountering random-like, low-correlation codewords. For this reason, it is widely recognized that in a TCMA system, a user-specific interleaver is required at the output of the TCM scheme in order to achieve a good Bit Error Ratio (BER) performance [2], since the interleaved codewords become more random-like and potentially impose a reduced interference owing to their lower correlation. Consequently,

The work reported in this paper has formed part of the Core 4 Research Programme of the Virtual Centre of Excellence in Mobile and Personal Communications, Mobile VCE, www.mobilevce.com, whose funding support, including that of the EPSRC, is gratefully acknowledged. Fully detailed technical reports on this research are available to Industrial Members of Mobile VCE. a TCMA system can be considered to be a special case of IDMA [3] employing TCM codes as the outer channel code and dispensing with the DS-spreading stage of IDMA.

On the other hand, IDMA can be viewed as chipinterleaved DS-CDMA that employs binary transmitted symbols for each user and is applicable for low-rate UpLink (UL) communications. It was also shown in [3] that the amalgamation of channel codes with IDMA systems and further enhanced by sophisticated power allocation is capable of approaching the channel's capacity. Thus it becomes evident that the family of pseudo-random LDPC codes, such as those proposed in [4], [5], constitutes particularly attractive component codes for CCDMA schemes, since they exhibit a near-capacity performance as well as being capable of differentiating the users, with the aid of their inherent interleavers. Despite these advantages, LDPC codeaided CCDMA may suffer from two potential drawbacks:

Memory inefficiency: Each user transmitting over the $Q$ user multiple access channel (MAC) is encoded as well as decoded by a channel code having a distinct parity-check matrix (PCM). This implies that a different PCM must be stored in a look-up table (LUT) for each user ${ }^{1}$. Therefore, the memory requirements are (linearly) dependent on both the LDPC code's block length and on the PCM parameters, as well as on the number of users supported by the system.

Unequal protection: If we assume having equal average transmit powers for each user and hence each user experiences the same inter-user interference, then the individual BER/BLER performance is only dependent on the channel code employed. However, when using LDPC codes having pseudo-random PCMs, it becomes quite difficult to construct a significantly high number of user-specific codes having identical graph-theoretical properties such as the girth, to maintain the same protection for each user.

In this paper, we consider a CCDMA system based on the recently proposed Multilevel Structured (MLS) LDPC codes [7]. Our novel contributions are:

- We circumvent the first problem of high memory requirements by taking advantage of the compact PCM description of Multilevel Structured (MLS) LDPC codes, which were recently proposed in [7];

- We ensure that each user's bits are equally protected and thus all users benefit from the same quality of service (QoS), which is achieved by utilizing isotopic,

\footnotetext{
${ }^{1}$ As an example, if each of the $Q$ PCMs has a column weight of $\gamma$ and a block length of $N$, then the LUT has to store the position of $Q N \gamma$ non-zero PCM entries, each representing an edge of the corresponding Tanner graph [6].
} 
user-specific (distinct) Latin squares.

To elaborate a little further, a $J$-level MLS code inherently possesses both pseudo-random as well as structured characteristics, and can be described by means of a base matrix, $J$ number of constituent matrices and a so-called adjacency matrix, where the latter can be represented by a Latin square. We will demonstrate that by using the same $J$ constituent matrices for each user, the memory requirements become practically independent of the total number of users present in the system, since the users may be uniquely identified with the aid of a user-specific $(J \times J)$-element Latin square. Furthermore, by exploiting isotopic Latin squares, we propose a technique of constructing channel codes that are distinct, whilst guaranteeing a similar attainable BER/BLER performance for each user. We will demonstrate that despite imposing a beneficial compact structure, no Bit Error Ratio (BER) or Block Error Ratio (BLER) performance degradation was imposed compared to the corresponding CCDMA system using pseudo-random LDPC codes for differentiating the users, albeit the latter impose high memory requirements.

The structure of this paper is as follows. Section III introduces the general model of the CCDMA system, whilst Section III outlines the construction of MLS codes. Then Section IV details the technique proposed for generating userspecific channel codes by exploiting the construction of MLS LDPC codes. Our simulation results are then presented in Section V. Finally, our conclusions are offered in Section VI.

\section{General Model of the CCDMA System}

Figure 1 depicts the general model of the CCDMA system, where the $q$ th user's signal $b_{q}$ is encoded by his/her userspecific channel code $C_{q}, q=1, \ldots, Q$, having a rate of $R$, resulting in the codeword $x_{q}=C_{q}\left(b_{q}\right)$. In a conventional IDMA system, the channel code may be the same for all users if a user-specific interleaver is employed, hence user $q$ will transmit the bit-stream of $x_{q}=\pi_{q}\left[C\left(b_{q}\right)\right]$ over the MAC. The canonical discrete-time real-valued model of the MAC seen in Figure 1 is then given by:

$$
y=\sum_{q=1}^{Q} h_{q} x_{q}+n,
$$

where $x_{q} \in\{ \pm 1\}, y$ and $n \sim \mathcal{N}\left(0, \sigma_{n}^{2}\right)$ denotes the transmitted signal, the received signal and the Additive White Gaussian Noise (AWGN), respectively. The parameter $h_{q}$ denotes the identical independently distributed (i.i.d.) UL Channel Impulse Response (CIR) of user $q$, whilst $\sigma_{n}^{2}$ represents the noise variance.

An iterative receiver, consisting of a Soft-In Soft-Out (SISO) detector and a bank of $Q$ individual SISO MLS LDPC decoders, is used for the sake of seeking a tradeoff between the higher performance and complexity of optimal joint detection and decoding as well as the performance loss of the lower-complexity separate detection and single-user LDPC decoding. Using the low-complexity Parallel Interference Cancellation (PIC) scheme introduced in [3], we can rewrite (1) as $y=h_{q} x_{q}+\xi$, where

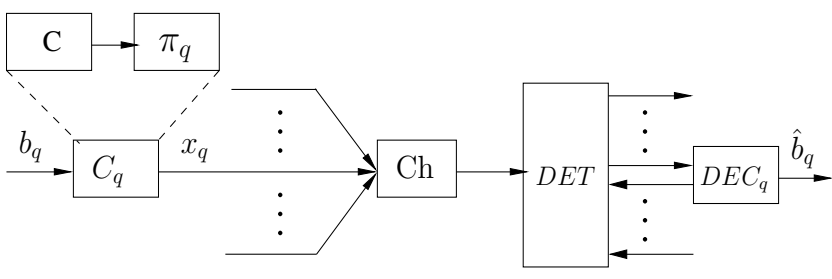

Fig. 1. A general simplified model for a purely channel coded IDMA-like channel code division multiple access (CCDMA) system.

$\xi=\sum_{j \neq q}^{Q} h_{j} x_{j}+n$ represents the interference plus noise. In the case of binary modulation, the real part $(R e)$ of $h_{q}^{*} y$ constitutes sufficient statistics for estimating $x_{q}$, resulting in $\operatorname{Re}\left(h_{q}^{*} y\right)=\left|h_{q}\right|^{2} x_{q}+\operatorname{Re}\left(h_{q}^{*} \xi\right)$, where $(\cdot)^{*}$ denotes the complex conjugate computation. We denote the soft estimate of a variable $a$ by $(\hat{a})$. Then, $\operatorname{Re}\left(h_{q}^{*} \hat{\xi}\right)$ and its variance $\mathbf{V}\left[\operatorname{Re}\left(h_{q}^{*} \hat{\xi}\right)\right]$ are formulated by:

$$
\begin{aligned}
\operatorname{Re}\left(h_{q}^{*} \hat{\xi}\right)= & h_{q}^{R e} \hat{y}^{R e}+h_{q}^{I m} \hat{y}^{I m}-\left|h_{q}\right|^{2} \hat{x}_{q}, \\
\mathbf{V}\left[\operatorname{Re}\left(h_{q}^{*} \hat{\xi}\right)\right]= & \left(h_{q}^{R e}\right)^{2} \mathbf{V}\left(\hat{y}^{R e}\right)+\left(h_{q}^{I m}\right)^{2} \mathbf{V}\left(\hat{y}^{I m}\right) \\
& -\left|h_{q}\right|^{4} \mathbf{V}\left(\hat{x}_{q}\right)+2 h_{q}^{R e} h_{q}^{I m} \phi,
\end{aligned}
$$

where $\phi=\sum_{q=1}^{Q} h_{q}^{R e} h_{q}^{\operatorname{Im}} \mathbf{V}\left(\hat{x}_{q}\right)$ and $\operatorname{Im}(\cdot)$ represents the imaginary part of a complex number. The soft estimate $\hat{y}$ and its variance can be expressed by:

$$
\begin{aligned}
\hat{y}^{R e} & =\sum_{q=1}^{Q} h_{q}^{R e} \hat{x}_{q} \\
\mathbf{V}\left(\hat{y}^{R e}\right) & =\sum_{q=1}^{Q}\left(h_{q}^{R e}\right)^{2} \mathbf{V}\left(\hat{x}_{q}\right)+\sigma_{n}^{2} .
\end{aligned}
$$

We remark that (4) and (5) would be equally valid for the imaginary counterpart. The soft bit $\hat{x}_{q}$ can be represented as $\hat{x}_{q}=\tanh \left[L_{d e c}^{e}\left(x_{q}\right) / 2\right]$, while its variance is given by $\mathbf{V}\left(\hat{x}_{q}\right)=1-\hat{x}_{q}^{2}$. Assuming that $\xi$ is Gaussian distributed, the extrinsic information $L_{d e t}^{e}\left(x_{q}\right)$ is given by:

$$
L_{\text {det }}^{e}\left(x_{q}\right)=2\left|h_{q}\right|^{2} \frac{\operatorname{Re}\left(h_{q}^{*} y\right)-\operatorname{Re}\left(h_{q}^{*} \hat{\xi}\right)}{\mathbf{V}\left[\operatorname{Re}\left(h_{q}^{*} \hat{\xi}\right)\right]} .
$$

Then, this extrinsic information gleaned from the detector is used as the a priori information input to the channel decoder, which computes a more reliable extrinsic information $L_{d e c}^{e}\left(x_{q}\right)$ for the next iteration. LDPC decoding was performed using the sum-product algorithm (SPA) [8], where messages are exchanged between the nodes residing at both sides of the corresponding Tanner graph.

\section{Construction OF MLS LDPC CODES AND THEIR LATIN SQUARE REPRESENTATION}

We consider MLS LDPC codes described by their regular bipartite graphs, $G(\mathbf{H})$, associated with a PCM $\mathbf{H}$ whose rows constitute the null space of the linear code $\mathbb{C}$ constructed over $\mathrm{GF}(2)$. Then, the graph $G(\mathbf{H})$ consists of the non-empty set of elements $\{V(G), C(G), E(G)\}$, where $V(G)=v_{1}, v_{2}, \ldots, v_{N}$ and $C(G)=c_{1}, c_{2}, \ldots, c_{M}$ represent the disjoint vertex-sets of the variable nodes and check nodes, 
whilst $E(G)$ is the set representing the edges. Furthermore, we assume that the degree of the variable nodes $v_{r} \in V(G)$, $r \in[1, N]$, is $\gamma$, and that of the check nodes $c_{s} \in C(G)$, $s \in[1, M]$, is $\rho$. Since the PCM of an MLS code $\mathbf{H}$ has a full rank, and a size of $(M \times N)$ elements, where the MLS LDPC code's block length is $N=|V(G)|$, the code rate becomes $R=1-M / N$. Each non-zero entry in $\mathbf{H}$ will then represent an edge of the corresponding Tanner graph.

The construction of a $J$-level MLS code requires a base matrix, a set of $J$ constituent matrices as well as an adjacency matrix [7]. The base matrix, hereby denoted by $\mathbf{H}^{b}$, is a sparse matrix defined over $\mathrm{GF}(2)$ having $\left(M^{b} \times N^{b}\right)$ elements, and contains exactly $\rho$ and $\gamma$ non-zero entries, randomly positioned in each of its rows and columns, respectively. The set of $J$ constituent matrices is then represented by $\boldsymbol{\Omega}=\left\{\mathbf{Q}_{0}, \mathbf{Q}_{1}, \ldots, \mathbf{Q}_{J-1}\right\}$, where the non-zero constituent matrix $\mathbf{Q}_{j}, j=0, \ldots, J-1$, is a distinct sparse matrix over $\mathrm{GF}(2)$ having the same dimensions as the base matrix. All the non-zero entries of all the $J$ sparse constituent matrices in $Q_{j} \in \Omega$ must occur in the same corresponding position of the non-zero entries of the base matrix ${ }^{2}$. Furthermore, a non-zero entry in a particular location of $\mathbf{Q}_{j} \in \boldsymbol{\Omega}$ implies that the entries in the corresponding locations of $\mathbf{Q}_{i} \in \boldsymbol{\Omega}$ are zero, where $i \in[0, J-1]$ and $i \neq j$.

Finally, we also define an adjacency matrix, which is a $(J \times J)$-element array matrix represented by $\mathbf{P}_{J}$, whose row (and column) blocks represent a sharply transitive set of $J$ permutations within $\Omega$. This implies that given any pair of constituent matrices $\mathbf{Q}_{x}, \mathbf{Q}_{y} \in \boldsymbol{\Omega}$, there exists a unique bijective mapping function $f: \boldsymbol{\Omega} \mapsto \boldsymbol{\Omega}$ in the set described by the row (and column) block of $\mathbf{P}_{J}$ that maps $\mathbf{Q}_{x} \in \boldsymbol{\Omega}$ to the image $\mathbf{Q}_{y}=f\left(\mathbf{Q}_{x}\right) \in \boldsymbol{\Omega}$. The adjacency matrix determines the position of each $\mathbf{Q}_{j} \in \boldsymbol{\Omega}$ with respect to the PCM $\mathbf{H}$ of the MLS code. From its definition, it follows that the adjacency matrix will not position any constituent matrix in the same row or column block, in order to achieve the required check and variable node distribution while at the same time ensuring that the girth of the corresponding Tanner graph is at least six.

From another point of view, it can be argued that the $J$ rows and columns of $\mathbf{P}_{J}$ represent a Latin square of order $J$, or equivalently, a 1-factorization of a bipartite graph. Consequently, a $J$-level MLS code can also be regarded as an edge-colored, complete bipartite graph of degree $J$. This brings us to the notion of what is known as coloring [9] of edges, where $E(\mathbf{H})$ is said to be an edge-coloring of $G(\mathbf{H})$ if any two edges on the graph containing the same vertex have different colors. Correspondingly, each symbol of the Latin square will create a monochromatic 1-factor of the Tanner graph. Figure 2 depicts the Latin square representation for a six-level MLS code, where in this case, its adjacency matrix is effectively a reduced Latin square. The $J$ rows and columns of the Latin square will then correspond to the respective

\footnotetext{
${ }^{2}$ Symmetrically repeated non-zero entries in two or more rows (or columns) in any of the matrices $\mathbf{Q}_{j} \in \boldsymbol{\Omega}, j=0, \ldots, J-1$, can be avoided by ensuring that the base matrix corresponds to a base protograph (refer to [7]) having a girth of at least six. Note furthermore that the position of the non-zero entries in each of the constituent matrices in $\mathbf{Q}_{j} \in \boldsymbol{\Omega}$ are also chosen at random.
}

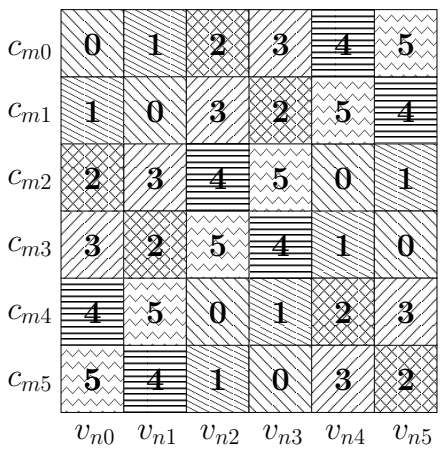

Fig. 2. A Latin square representation of the adjacency matrix of a six-level MLS code. The $J$ rows and columns of the Latin square correspond to the respective multi-check node $c_{m j}$ and multi-variable node $v_{n j}$, where $m=1, \ldots, M^{b}, n=1, \ldots, N^{b}$ and $j=0, \ldots, J-1$.

multi-check node $c_{m j} \subset C(\mathbf{H})$ and to the multi-variable node $v_{n j} \subset V(\mathbf{H}),\left|c_{m j}\right|=\left|v_{n j}\right|$, where we have $m=1, \ldots, M^{b}$, $n=1, \ldots, N^{b}$ and $j=0, \ldots, J-1$. Each symbol in the Latin square will then correspond to a multi-edge with a different edge-color on the corresponding graph.

\section{Generating User-Specific Channel Codes USING MLS LDPC CODES}

In this light, two seemingly contradictory problems must be outlined. Firstly, since the $Q$ users are being separated in the MLS LDPC code domain, a user-specific channel code is required. This makes the memory requirements at the transceiver dependent on the number of users present in the system, which is undesirable in memory-constrained hand-held transceivers. Secondly, each of the $Q$ user must be guaranteed the same BER/BLER performance at any signalto-noise ratio (SNR). Therefore, the channel code must be distinct, whilst at the same time guarantee a similar attainable BER/BLER performance for each user. These two problems are tackled separately in the forthcoming subsections.

\section{A. User Separation by Distinct Latin Squares}

We reduce the memory requirements by using the same base matrix and $J$ constituent matrices for all the $Q$ users in the system. This implies that the receiver will only have $J$ distinct memory blocks, each corresponding to a constituent matrix having a dimension, which is a factor of $1 / J$ lower than that of a single PCM. However, a distinct adjacency matrix is then allocated for each user, and hence the required user separation is achieved by assigning a different Latin square to each of the $Q$ users. The number of distinct Latin squares of order $J$ is given by $\mathcal{X}_{\mathcal{J}}=J ! \times(J-1) ! \times L(J, J)[10]$, where $L(J, J)$ is the number of normalized $(J \times J)$-element Latin squares.

For the sake of simplifying our analysis, let us consider the simple example of a six-level MLS code. The total number of Latin squares of order six is equal to $\mathcal{X}_{6}=6 ! \times 5 ! \times 9408$ [10]. This means that using a six-level MLS code, we can describe a total of $\mathcal{X}_{6}$ unique PCMs, corresponding to $\mathcal{X}_{6}$ unique Tanner graphs, and thus representing a total of $\mathcal{X}_{6}$ unique binary codes, whilst still sharing the same base matrix and requiring a total of only six constituent matrices for differentiating the $Q$ users. Therefore, a CCDMA system employing six-level MLS 
codes can potentially distinguish between a total of $\mathcal{X}_{6}$ users by only storing six $\left(M^{b} \times N^{b}\right)$-element constituent matrices and $Q$ adjacency matrices, where we have $M^{b}=M / J$ and $N^{b}=N / J$. The dimension of an adjacency matrix is only $(J \times J)$, where $J$ is much smaller than both $M$ and $N$, therefore its storage requirements can be considered to be negligible when compared to the $(M \times N)$-element PCM. Therefore, our proposed system renders the memory requirements practically independent of the total number of users supported by the system. On the other hand, any other LDPC code-aided CCDMA system has to store $Q$ PCMs, each having a dimension of $(M \times N)$, thus requiring in total the enumeration of $Q N \gamma$ number of edges.

\section{B. Isotopic Latin Squares and Isomorphic Edge-Colored Graphs}

This subsection outlines the technique that was employed in order to ensure that all the $Q$ users benefit from the same QoS. This brings us to the notion of isotopic Latin squares and isomorphic edge-colored graphs. Two Latin squares $S$ and $S^{\prime}$ are said to be isotopic, if there exists a triple $(\alpha, \beta, \chi)$ (referred to as an isotopy $^{3}$, where $\alpha, \beta$ and $\chi$ correspond to a row, column and symbol permutation, respectively, which carries the Latin square $S$ to $S^{\prime}$. Effectively, this implies that if we consider any particular row and column position of the Latin square specified by the check nodes as well as variable nodes $\left(c_{m j}, v_{n j}\right)$ and containing the entry $e$, where $m=1, \ldots, M^{b}, n=1, \ldots, N^{b}$ and $j=0, \ldots, J-1$, the entry at position $\left(\alpha\left(c_{m j}\right), \beta\left(v_{n j}\right)\right)$ of the Latin square $S^{\prime}$ will be equal to $\chi(e)$. Two Latin squares $S_{1}$ and $S_{2}$ will then give rise to isomorphic edge-colored complete bipartite graphs if and only if Latin square $S_{1}$ is isotopic to either another Latin square $S_{2}$ or to its transpose [12].

Since the decoding of LDPC codes is very much dependent on their graph-theoretic properties, we can ensure the same QoS for each user, if all the user-specific channel codes $C_{q}$, $q=1, \ldots, Q$, have the corresponding edge-colored Tanner graphs that exhibit identical graph-theoretic properties, and thus are isomorphic. This can be achieved by allocating adjacency matrices to the $Q$ users that are represented by both distinct as well as isotopic Latin squares.

\section{Simulation Results}

The results presented in this section were obtained using Binary Phase Shift Keying (BPSK) modulation, when transmitting over the AWGN as well as uncorrelated Rayleigh (UR) multiple access channels and using LDPC code-aided CCDMA systems in conjunction with both sixlevel MLS codes [7] as well as pseudo-random MacKay [13] codes. We have considered half-rate LDPC codes having $\gamma=3$ and a block length of $N=1008$ bits. The number of users supported by the system was $Q=3$, and therefore the bandwidth efficiency defined as $R Q$ was $1.5 \mathrm{bps} / \mathrm{Hz}$. The number of iterations between the PIC detector and the LDPC

\footnotetext{
${ }^{3}$ Then, an isotopy class comprises the set of all the Latin squares isotopic to a given Latin square. A list of the isotopy classes for Latin squares of small orders is given by McKay in [11].
}

decoder was set to $I=5$ for $Q=2$ users and $I=10$ for $Q=3$ users. The LDPC decoding was performed using the SPA having a maximum of 100 iterations.

Figure 3 illustrates the BER and BLER performance comparison of a CCDMA scheme using half-rate MacKay and six-level MLS LDPC codes having a block length of $N=1008$, when transmitting over the AWGN and UR channels. In Figures 3(a) and 3(b), we compare the achievable BER as well as the BLER performance using the $N=1008$ MacKay and MLS codes as component codes, and a userspecific pseudo-random interleaver after the channel encoder. It can be observed that the BER/BLER performance of both systems is comparable. We point out that in this case there is no need for a distinct code description $C_{q}$ for each user, $q \in[1, Q]$, since the LDPC encoded bit stream of each user is interleaved by a user-specific interleaver before being transmitted over the multiple access channel. Our motivation of showing the results in Figures 3(a) and 3(b) is to explicitly demonstrate that both systems have a similar performance.

We then proceed to remove the user-specific interleaver, when user separation is then entirely achieved by the distinct (and isotopic) Latin squares. The BER and BLER performance exhibited in this scenario is shown in Figures 3(c) and 3(d), where we compared the performance of the MLS LDPC coded CCDMA system both with and without the interleaver. Once again, the proposed system does not suffer from any BER/BLER performance loss.

However, the proposed system has considerable gains in terms of the interleaver storage and delay requirements, since there is no need to store user-specific interleavers or a userspecific PCM. For the case of the benchmarker system using the pseudo-random MacKay codes, the memory LUT must store the location of 9072 edges in order to fully describe the three distinct PCMs. On the other hand, the IDMA system using MLS LDPC codes as component codes is more memoryefficient, since in this case the LUT has to enumerate only 612 edges in order to store the six distinct $(84 \times 168)$-element constituent matrices and the three $(6 \times 6)$-component Latin squares (adjacency matrices). Furthermore, we note that the difference in the memory requirements of the two systems will become more pronounced upon increasing the number of users $Q$ or the block length $N$. The proposed system will be applicable in situations, where low-delay requirements are an absolute necessity, for example in interactive, lip-synchronized speech and video communications.

\section{SUMMARY AND CONCLUSIONS}

The concept of CCDMA was proposed, arguing that an LDPC code-aided CCDMA system is generally inapplicable in memory-constrained scenarios, since a distinct PCM code description is required for each user, which has to be stored in memory, in order to be able to differentiate each user. In this paper, we have proposed a specific instantiation of a CCDMA system using MLS LDPC codes, where we exploited the compactness of the MLS LDPC code description in order to significantly reduce the memory requirements. By using the same $J$ constituent matrices for each user, we 


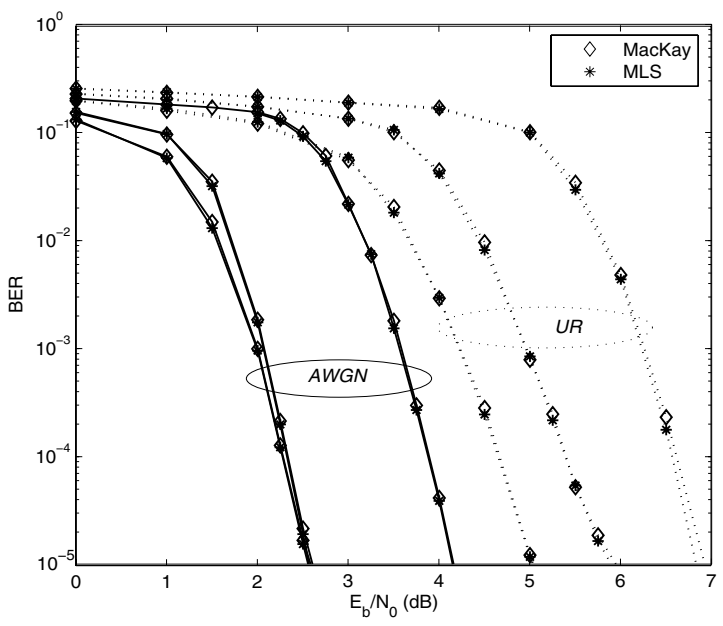

(a) BER, MLS and MacKay with interleaver

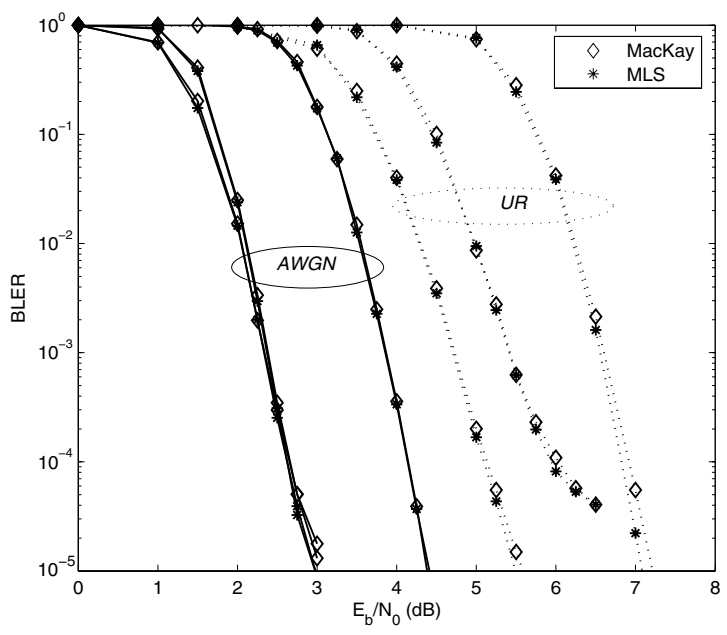

(b) BLER, MLS and MacKay with interleaver

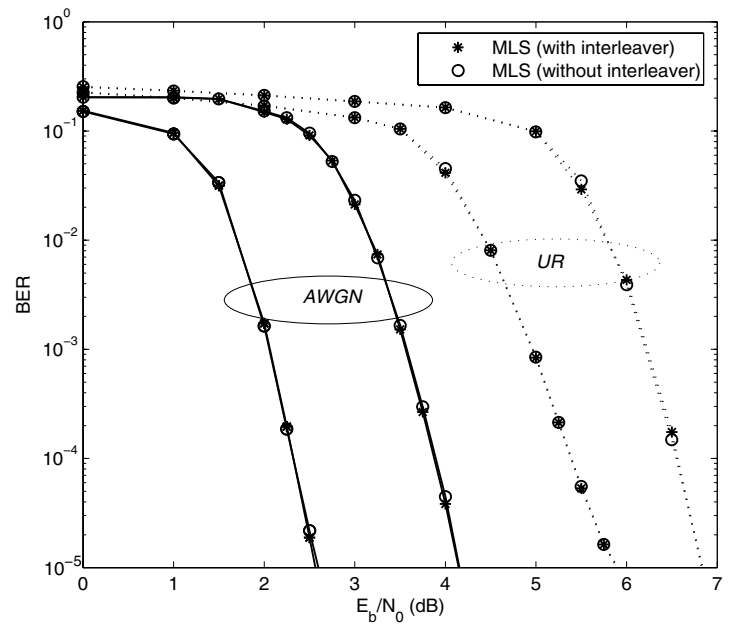

(c) BER, MLS with/without interleaver

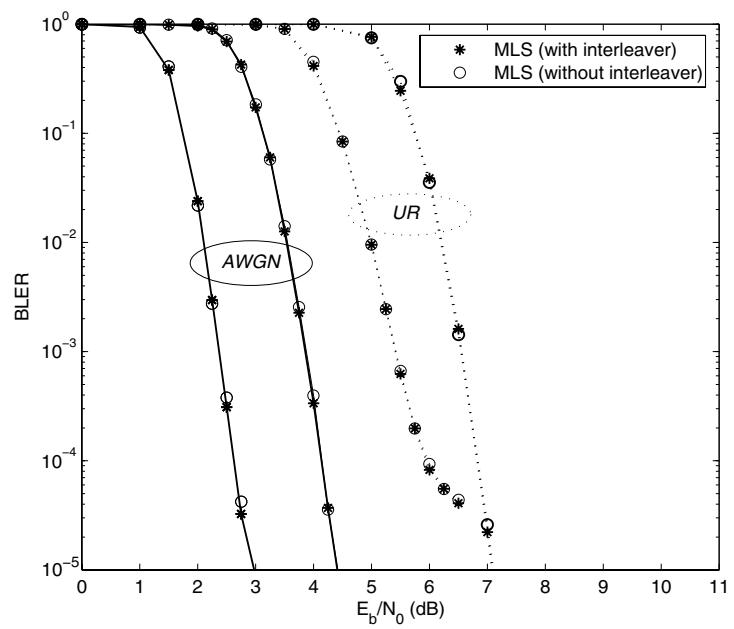

(d) BLER, MLS with/without interleaver

Fig. 3. A BER and BLER performance comparison of a purely channel coded IDMA using half-rate MacKay [13] and six-level MLS LDPC codes having a block length of $N$ when transmitting over the AWGN and UR channels. (a) and (b): A comparison of the BER and BLER performance of the IDMA system using MacKay and MLS codes, where both systems also have a user-specific interleaver, for 1, 2 and 3 users. (c) and (d): A comparison of the BER and BLER performance for 2 and 3 users of the IDMA system using MLS codes with as well as without the user-specific interleaver.

succeeded in rendering the memory requirements practically independent of the total number of users present in the system, since each user is only distinguished by means of a different $(J \times J)$-component Latin square instead of a different PCM. Furthermore, we outlined a technique based on isotopic Latin squares that makes it possible to construct channel codes that are distinct, whilst guaranteeing a similar attainable BER/BLER performance for each user. We demonstrated that these advantages accrue without any compromise in the attainable BER/BLER performance, when compared to the corresponding pseudo-random LDPC based CCDMA benchmarker, which imposes significantly higher memory requirements. Our scheme is attractive in interactive, low-delay speech and video applications and is equally applicable for other classes of random-like codes such as Repeat-Accumulate (RA) codes.

\section{REFERENCES}

[1] L. Hanzo, L.-L. Yang, E.-L. Kuan, and K. Yen, Single- and MultiCarrier DS-CDMA: Multi-User Detection, Space-Time Spreading, Synchronisation, Networking and Standards. Wiley-IEEE Press, 2003.
[2] F. Brannstrom, T. Aulin, and L. Rasmussen, "Iterative detectors for trellis-code multiple-access," IEEE Transactions on Communications, vol. 50, pp. 1478-1485, Sept. 2002.

[3] L. Ping, L. Liu, K. Wu, and L. W. K., "Interleave-division multipleaccess," IEEE Transactions on Wireless Communications, vol. 5, pp. 938-947, Apr. 2006.

[4] R. G. Gallager, "Low-density parity-check codes," IRE Transactions Information Theory, vol. 45, pp. 21-28, Jan. 1962.

[5] D. MacKay, "Good error-correcting codes based on very sparse matrices," IEEE Transactions on Information Theory, vol. 45, pp. 399431, Mar. 1999.

[6] R. Tanner, "A recursive approach to low complexity codes," IEEE Transactions on Information Theory, vol. 27, pp. 533-547, Sept. 1981.

[7] N. Bonello, S. Chen, and L. Hanzo, "Multilevel structured LDPC codes,' in Proc. of IEEE ICC 'O8.

[8] F. Kschischang, B. Frey, and H.-A. Loeliger, "Factor graphs and the sum-product algorithm," IEEE Transactions on Information Theory, vol. 47, pp. 498-519, Feb. 2001.

[9] N. L. Biggs, Discrete Mathematics (2 ${ }^{\text {nd }}$ Edition). UK: Oxford University Press, Dec. 2002.

[10] B. D. McKay and E. Rogoyski, "Latin squares of order 10," The Electronic Journal of Combinatorics, vol. 2, 1995.

[11] B. D. McKay, "Latin squares," Available online from http://cs.anu.edu.au/ bdm/data/latin.html.

[12] R. A. Bailey and P. J. Cameron, "Latin squares: Equivalents and equivalence," Available online from http://designtheory.org/.

[13] D. MacKay, "Online database of low-density parity-check codes," Available online from wol.ra.phy.cam.ac.uk/mackay/codes/data.html. 